\title{
Crystal structure of PHD domain of UHRF1 and insights into recognition of unmodified histone $\mathrm{H} 3$ arginine residue 2
}

Cell Research (2011) 21:1374-1378. doi:10.1038/cr.2011.124; published online 2 August 2011

\section{Dear Editor,}

UHRF1 (ubiquitin-like, containing plant homeodomain (PHD) and RING finger domains, 1), also known as ICBP90 in human and NP95 in mouse, is a critical regulator of maintenance of $\mathrm{CpG}$ DNA methylation through targeting de novo DNA methyltransferase (DNMT1) to hemimethylated replication forks; its ablation leads to genomic hypomethylation and cell cycle arrest $[1,2]$. UHRF1 is also involved in heterochromatin formation [3], and in silencing tumor suppressor genes, such as RB1 and $\mathrm{p} 16^{\mathrm{INK} 4 \mathrm{~A}}$. It has been reported that UHRF1 is associated with DNMT1 [1, 2], H3K9 methyltransferase (G9a), histone deacetylase 1 and histone acetyltransferase (Tip60). UHRF1 is composed of at least five recognizable protein modules (Figure 1A), including the SET and RING associated (SRA) domain, which binds methyl cytosine $[1,2]$, and a tandem Tudor domain, which binds trimethylated histone $\mathrm{H} 3$ lysine 9 (H3K9me3) [4]. Both epigenetic modifications are hallmarks of peri-centromeric heterochromatin $(\mathrm{PCH})$ [5-7], where UHRF1 is preferentially localized. The PHD of UHRF1 has been reported to be involved in large-scale reorganization of PCH [8], and together with the SRA domain, define the binding affinity to $\mathrm{H} 3 \mathrm{~K} 9 \mathrm{me} 3$ [3]. However, recent studies show that $\mathrm{H} 3 \mathrm{~K} 9 \mathrm{me} 3$ binding is mediated by the tandem Tudor domain $[9,10]$. Thus, what PHD domain binds to remains unclear.

To elucidate the binding specificity of the PHD domain of UHRF1 for histone H3 modification, we initially carried out isothermal titration calorimetry (ITC) assays using purified $\mathrm{PHD}_{\mathrm{UHRF}}$ (residues 311-379) and various histone $\mathrm{H} 3$ peptides with or without modifications (Supplementary information, Table S1). As shown in Figure $1 \mathrm{~B}, \mathrm{PHD}_{\mathrm{UHRF}}$ specifically and robustly $(K \mathrm{~d}=0.93$ $\mu \mathrm{M}$ ) binds the unmodified, N-terminal histone $\mathrm{H} 3$ tail (aa 1-12). $\mathrm{PHD}_{\mathrm{UHRF}}$ also binds the $\mathrm{H} 3 \mathrm{~K} 9 \mathrm{me} 3$ peptide, with a binding affinity $(\mathrm{Kd})$ of $0.69 \mu \mathrm{M}$, which is comparable to that of $\mathrm{PHD}_{\mathrm{UHRF} 1}$ for unmodified $\mathrm{H} 3$ tail, suggesting that trimethylated $\mathrm{H} 3 \mathrm{~K} 9$ may not be involved in $\mathrm{PHD}_{\mathrm{UHRF}^{-}}$ mediated histone tail recognition. Tri-methylation at
H3K4 reduced binding affinity by approximately threefold, suggesting that H3K4 may not be involved in or plays a minor role for the specific interaction with $\mathrm{PHD}_{\mathrm{UHRF}}$. In contrast, ITC results (Figure 1B, Supplementary information, Data S1 and Figure S1) show that monomethylation of H3R2 reduced the binding affinity for $\mathrm{PHD}_{\mathrm{UHRF}}$ by approximately six fold, suggesting an important role of $\mathrm{H} 3 \mathrm{R} 2$ residue for $\mathrm{PHD}_{\mathrm{UHRF}}$ interaction. More importantly, asymmetric or symmetric dimethylation of H3R2 has the most significant impact on binding ( $K \mathrm{~d}$ from $0.93 \mu \mathrm{M}$ to 17.4 and $14.5 \mu \mathrm{M}$, respectively, representing 19- and 16-fold reduction). Changing the residue $\mathrm{R} 2$ of $\mathrm{H} 3$ peptide to alanine also significantly reduced the binding affinity to $\mathrm{PHD}_{\mathrm{UHRF}}$, further demonstrating the critical role of $\mathrm{H} 3 \mathrm{R} 2$ for $\mathrm{PHD}_{\mathrm{UHRF}}$ interaction. These findings suggest that among the various histone $\mathrm{H} 3$ modifications, $\mathrm{PHD}_{\mathrm{UHRF} 1}$ binds unmodified $\mathrm{H} 3$, possibly via recognition of the unmodified $\mathrm{R} 2$ residue.

To test whether other domains of UHRF1 also bind unmodified histone $\mathrm{H} 3$, we performed ITC analyses using unmodified $\mathrm{H} 3$ titrating the tandem Tudor domain and SRA domain, both of which are important protein modules of UHRF1 for recognition of epigenetic modifications. The ITC results show that Tudor $_{\text {UHRF1 }}$, which mainly recognizes $\mathrm{H} 3 \mathrm{~K} 9 \mathrm{me} 3$ [9], had significantly less affinity for the unmodified $\mathrm{H} 3(K \mathrm{~d}=35.8 \mu \mathrm{M})$. The SRA domain, which binds hemimethylated DNA, had no detectable binding to histone H3 (Supplementary information, Figure S2 and Table S2). These findings indicate that among the various protein modalities present in UHRF1 (Figure 1A), $\mathrm{PHD}_{\mathrm{UHRF}}$ is responsible for binding unmodified $\mathrm{H} 3$.

To understand the molecular mechanism of $\mathrm{PHD}_{\mathrm{UHRF}^{-}}{ }^{-}$ mediated histone $\mathrm{H} 3$ recognition, we determined the crystal structure of $\mathrm{PHD}_{\mathrm{UHRF}}$ in complex with unmodified $\mathrm{H} 3$ peptide at $1.8 \mathrm{~A}$ resolution. The statistics for the structure determination are summarized in Supplementary information, Table S3. Interestingly, the PHD $_{\text {UHRF1 }}$ forms a dimer in crystal, which is mediated by a zinc atom coordinated by residues E375, H332 from both monomers (Supplementary information, Data S1 and 

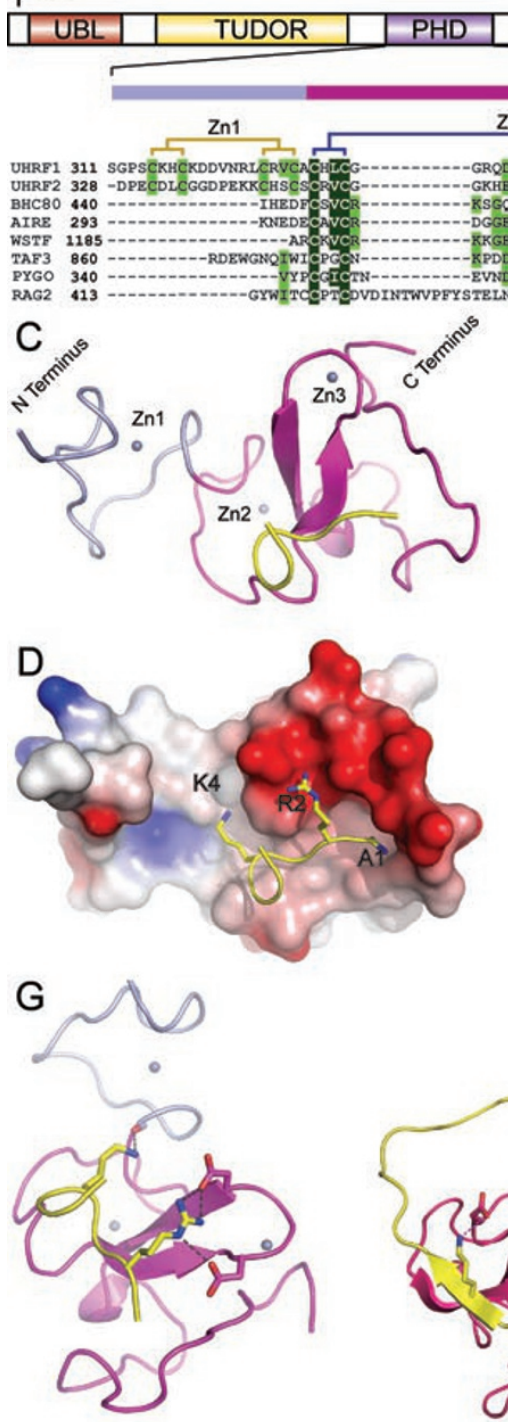

PHDUhrF1 + H3

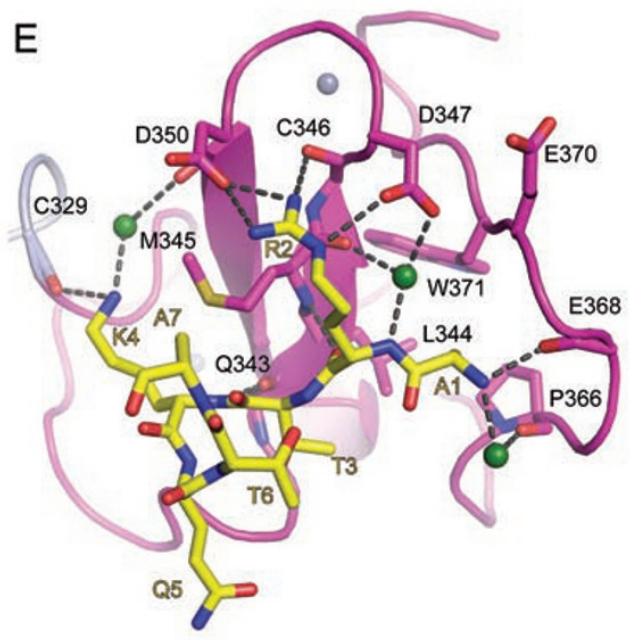

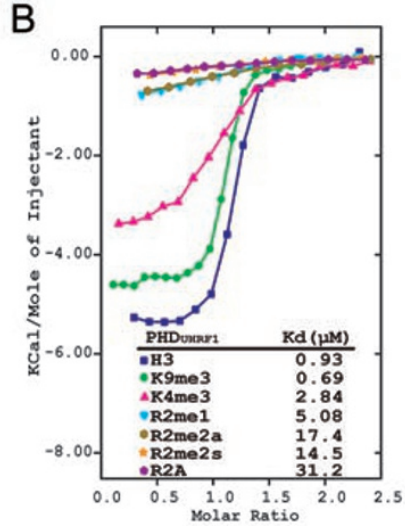
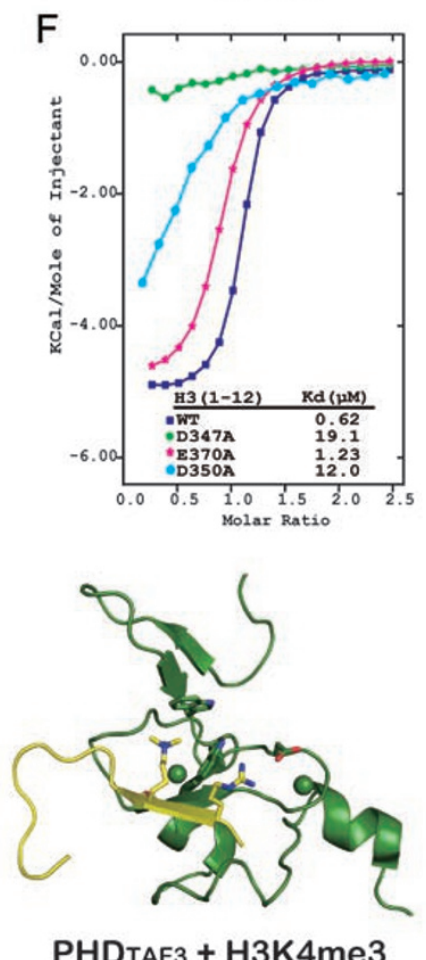

PHDTAF3 + H3K4me3
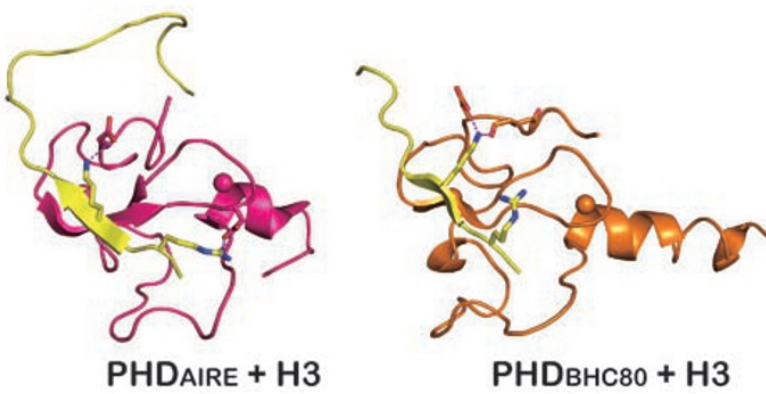

Figure 1 The UHRF1 PHD domain recognizes unmodified histone H3 tail. (A) Schematic representation of domain structure of human UHRF1. Structure-based sequence alignment of PHD $\mathrm{UHRF}_{1}$ with other representative PHD domains is indicated with secondary structural elements shown above the sequences. (B) Superimposed ITC enthalpy plots for the binding of various histone $\mathrm{H} 3$ peptides with or without modifications (syringe) to $\mathrm{PHD}_{\mathrm{UHRF}}$ (cell) with the estimated binding affinity $(\mathrm{Kd})$ listed. (C) Crystal structure of $\mathrm{PHD}_{\mathrm{UHRF}}$ in complex with unmodified histone $\mathrm{H} 3$ peptide shown as ribbon representation. The first zincfinger is colored in light blue, and the second and third zinc-fingers are colored in purple. Histone $\mathrm{H} 3$ peptide is colored in yellow and three zinc atoms are shown in gray balls. (D) PHD $\mathrm{UHRF}_{1}$ is shown as surface representation with electrostatic potential and $\mathrm{H} 3$ peptide is shown as ribbon representation and colored in yellow. Critical residues involved in the interaction (A1, R2 and K4) are shown as sticks representation. The complex structure is orientated as in $\mathbf{C}$. (E) Detailed interaction between $\mathrm{PHD}_{\mathrm{UHRF} 1}$ and $\mathrm{H} 3$ peptide with critical residues involved in the interaction shown as sticks representation and hydrogen bonds as black dashed lines. (F) Superimposed ITC enthalpy plots for the binding of unmodified histone $\mathrm{H} 3$ peptide (syringe) to wild-type or mutants of $\mathrm{PHD}_{\mathrm{UHRF} 1}$ (cell) with the estimated binding affinity $(K d)$ listed. (G) Structure comparison of $\mathrm{PHD}_{\mathrm{UHRF}}: \mathrm{H}_{3}$ complex with other PHD:H3 complexes. Similarity search was performed with the Dali server and representative structures

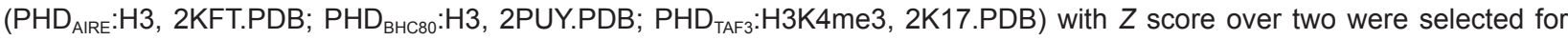
structure comparison. Ribbon representations are shown with $\mathrm{H} 3$ peptide colored in yellow, and the side chains of residues for specific recognition shown as stick representation and hydrogen bonds indicated as dashed lines. 
Figure S3). The dimer interface is away from the histone $\mathrm{H} 3$ peptide binding surface. Thus, we will not discuss the dimer formation in this report. $\mathrm{PHD}_{\mathrm{UHRF} 1}$ monomer is coordinated by three zinc atoms and forms a rodshape structure, consisting of a small $\alpha$ helix, a doublestranded anti-parallel $\beta$-sheet and three loops (Figure $1 \mathrm{C}$ and Supplementary information, Figure S4). The C terminus of $\mathrm{PHD}_{\mathrm{UHRF}}$ (purple) is coordinated by two zinc atoms in an interleaved manner (Figure $1 \mathrm{C}$ and Supplementary information, Figure S4), which is similar to the previously reported histone tail-binding PHD domain structures, including BPTF, ING2, TAF3, BHC80, AIRE, PYGO and RAG2. However, a difference lies at the $\mathrm{N}$ terminus of $\mathrm{PHD}_{\mathrm{UHRF}}$ (light blue), where the first zinc atom coordinates the N-terminal loop region. Such a structural feature was not found for the other histonebinding PHD domains, although the function of the first zinc-finger remains to be determined (Figure 1A). The histone peptide adopts a coil conformation (yellow) with the $\mathrm{N}$-terminal residues (A1 to K4) positioned on the surface of $\mathrm{PHD}_{\mathrm{UHRF}}$. Residues Q5 to A7 of H3, which form a short turn, were also clearly traced in the structure model and have no direct interaction with $\mathrm{PHD}_{\mathrm{UHRF}}$. The $\mathrm{C}$ terminus of $\mathrm{H} 3$ peptide was not built in the final model because of lacking of electron density, which may result from high flexibility (Figure 1D). The major intermolecular interactions involve the second and third zinc-finger of $\mathrm{PHD}_{\mathrm{UHRF}}$, while the first zinc-finger makes no contact with the histone peptide (Figure 1C and 1D).

Differing from other known PHD:H3 complexes, the H3 peptide does not adopt $\beta$-strand conformation and make characteristic $\beta$-sheet interactions with the two $\beta$ strands of $\mathrm{PHD}_{\mathrm{UHRF}}$. Two inter-main chain hydrogen bonds are formed between the $\mathrm{H} 3$ peptide and $\beta 2$ strand of $\mathrm{PHD}_{\mathrm{UHRF}}$, and an additional inter-main chain hydrogen bond is mediated by a water molecule (Figure 1E). The $\mathrm{N}$ terminus of histone $\mathrm{H} 3$ peptide is anchored into an acidic pocket through formation of hydrogen bonds with backbone carbonyl oxygen atoms of residues E368, and P366 (mediated by a water molecule) of $\mathrm{PHD}_{\mathrm{UHRF}}$. The $\mathrm{N}$ terminus is further stabilized with the methylene group of H3A1 facing toward the hydrophobic pocket formed by the side chains of L344, P366 and W371 of PHD $\mathrm{PHRF}_{\mathrm{UHR}}$. Intriguingly, the side chain of H3R2 forms four hydrogen bonds with the side chains of residues D347, D350, and the carbonyl group of C346 of $\mathrm{PHD}_{\mathrm{UHRF}}$ (Figure 1E), which support H3R2 as a major contact site for $\mathrm{PHD}_{\mathrm{UHRF}}$ recognition. Consistently, the electrostatic potential surface of $\mathrm{PHD}_{\mathrm{UHRF}}$ shows that H3R2 is projected onto the acidic surface of $\mathrm{PHD}_{\mathrm{UHRF}}$ (Figure 1D). The side chain of $\mathrm{H} 3 \mathrm{~K} 4$ forms hydrogen bonds with the carbonyl groups of residues C329, and D350 (mediated by a water molecule) of $\mathrm{PHD}_{\mathrm{UHRF}}$ (Figure 1E). Collectively, these results indicate that $\mathrm{H} 3 \mathrm{~A} 1$ and $\mathrm{H} 3 \mathrm{R} 2$ play a major role in $\mathrm{PHD}_{\mathrm{UHRF}}$-mediated histone $\mathrm{H} 3$ recognition, which may also contribute to the H3R2 specificity of this recognition.

The differential effects of various histone H3 modifications on $\mathrm{PHD}_{\mathrm{UHRF}}$ interaction can be explained based on the analyses of complex structure. Asymmetric or symmetric dimethylation of H3R2, which has been shown to significantly reduce the binding affinity $(\sim 16$ to 19 -fold) to PHD $_{\text {UHRF1 }}$ by ITC measurement, is expected to disrupt hydrogen bonds between guanidine group of H3R2 and residue D350 of PHD UHRF1 (for H3R2me2a), or residues D350, C346 of $\mathrm{PHD}_{\mathrm{UHRF} 1}$ (for H3R2me2s). The protruding methyl groups of H3R2me 2 may also occlude the interaction with residues D350, M345 and C346 of $\mathrm{PHD}_{\mathrm{UHRF}}$ because of steric hindrance, and further disrupt the hydrogen bond between H3R2 and residue D347 of $\mathrm{PHD}_{\mathrm{UHRF}}$, while monomethylation of H3R2 may have less effect of steric hindrance and thus leads to a less affinity reduction (approximately six fold) for $\mathrm{PHD}_{\mathrm{UHRF}}$ comparing to that of dimethylation of H3R2. In contrast, methylation of $\mathrm{H} 3 \mathrm{~K} 4$ had only three fold reduction of the binding affinity for $\mathrm{PHD}_{\mathrm{UHRF}}$ because residue $\mathrm{H} 3 \mathrm{~K} 4$ plays a minor role for the interaction and disruption of H3K4 binding will not largely affect the binding affinity.

We next performed ITC measurements to investigate amino acids in $\mathrm{PHD}_{\mathrm{UHRF}}$ that are involved in the interaction (Supplementary information, Table S4). As shown in Figure $1 \mathrm{~F}$, changing the $\mathrm{PHD}_{\mathrm{UHRF}} \mathrm{H} 3 \mathrm{R} 2$-contacting residue D347 to A significantly reduced the binding affinity ( $\sim 30$-fold reduction), indicating a critical role of the interaction between residue $\mathrm{D} 347$ of $\mathrm{PHD}_{\mathrm{UHRF}}$ and $\mathrm{R} 2$ of H3. Mutation of D350A also significantly decreased the binding affinity to $\mathrm{H} 3$ peptide ( 20-fold reduction). However, as a negative control, mutation of E370A only slightly (approximately two fold) reduced the binding affinity to $\mathrm{H} 3$ peptide, which may result from the conformational changes of the $\mathrm{H} 3$ binding interface since E370 is close to residue D347 in the three-dimensional structure. The results indicate that residues D347, D350, which are involved in H3R2 interaction, are important for $\mathrm{H} 3$ recognition, further supporting a critical role of H3R2 in the binding of the $\mathrm{PHD}_{\mathrm{UHRF}}$ domain to unmodified histone $\mathrm{H} 3$.

We further performed a peptide-binding assay using biotinylated histone $\mathrm{H} 3$ peptides with various methylation to pull-down full-length UHRF1 (Supplementary information, Figure S1C-S1D). The results show that UHRF1 binds to unmodified H3 and such interaction was significantly impeded by point mutation D347A/ E348, indicating that the PHD domain contributes to the 
specific interaction. We also found that UHRF1 binds to $\mathrm{H} 3 \mathrm{~K} 9 \mathrm{me} 3$, with stronger binding affinity than that of unmodified $\mathrm{H} 3$ peptide, which is consistent with the finding that tandem Tudor domain binds to $\mathrm{H} 3 \mathrm{~K} 9 \mathrm{me} 3$. Thus, UHRF1 specifically recognizes H3R2 residue with PHD and K9me3 with tandem Tudor domain. How the two domains recognize $\mathrm{H} 3$ tail modification coordinately remains to be further investigated.

Previous work identified a number of PHD domains that bind either methylated or unmethylated lysine residues on histone H3. Importantly, the above biochemical and structural analyses, together with the quantitative binding assays, identified a new histone tail recognition mechanism that is driven by binding to an unmodified arginine residue, H3R2. Comparison with the representative histone tail-binding PHD domains shows similar overall structures (Figure 1G). Interestingly, the PHD domains that recognize trimethylated H3K4 (TAF3) are characterized by an aromatic cage while binding of unmethylated $\mathrm{H} 3$ by the PHD domains of BHC 80 and AIRE is mediated by hydrogen bonds. For both type of PHD:H3 interactions, the $\mathrm{H} 3$ peptides adopt an extended $\beta$-strand conformation and form an anti-parallel $\beta$ sheet with a two-stranded $\beta$ sheet in PHD, with four to five intermolecular hydrogen bonds formed. However, in the $\mathrm{PHD}_{\mathrm{UHRF}}: \mathrm{H} 3$ complex structure, only two such hydrogen bonds and no intermolecular $\beta$-sheet formation were found. H3K4 methylation significantly affects the interaction between $\mathrm{BHC} 80$ and histone $\mathrm{H} 3$ peptide because the lysine 4 mainly contributes to the interaction, while for PHD ${ }^{\mathrm{UHRF} 1}$, arginine 2 methyaltion has a similar effect. The interaction is mainly mediated by hydrogen bonds between the side chain of H3R2 and acidic residues of $\mathrm{PHD}_{\mathrm{UHRF}}$. In this regard, the H3R2 recognition of $\mathrm{PH}-$ $\mathrm{D}_{\mathrm{UHRF}}$ mediated predominantly by inter-side chain hydrogen bonds represents a new mode of histone $\mathrm{H} 3$ tail recognition.

In summary, we have provided biochemical and structural data highlighting the discovery that the PHD domain of UHRF1 is an epigenetic regulatory module dedicated to the recognition of an unmodified arginine residue (R2) on histone H3. Together with the recent report of the first methylated arginine effector TDRD3, these findings begin to uncover a potentially elaborate effector network for the recognition of differential methylation states on histone arginine residues. The results also indicate that UHRF1 contains three modules, tandem Tudor, PHD and SRA domains, which specifically recognize three different modifications, H3K9me3, H3R2 and hemimethylated CpG DNA, respectively. Thus, in addition to being an E3 ubiquitin ligase and binding partner of epigenetic regulators, including DNMT1, G9a, HDAC and Tip60, UHRF1 may also function as a reader of multiple epigenetic modifications in vivo and mediate a possible crosstalk between specific modifications.

\section{Accession number}

The atomic coordinates of the $\mathrm{PHD}_{\mathrm{UHRF}}$ have been deposited in the Protein Data Bank with accession code 3SHB.

\section{Acknowledgments}

We thank staff members of beamline BL17U at SSRF (China) and Dr Yuhui Dong at BSRF (China) for the help in data collection and structure determination. This work was supported by grants from the National Basic Research Program of China (2009CB918600, 2011CB965300), the National Natural Science Foundation of China (30870493, 31030019, 11079016, 31000325), International Collaboration Program from Science and Technology Commission of Shanghai Municipality (10430709300) and Fok Ying Tung Education Foundation (20090071220012).

\section{Lulu $\mathrm{Hu}^{1,2, *}$, Ze Li ${ }^{2, *}$, Ping Wang ${ }^{2}$, Yan Lin ${ }^{2}$,} Yanhui $\mathrm{Xu}^{1,2,3}$

${ }^{I}$ Cancer Institute, Shanghai Cancer Center, Fudan University; Department of Oncology, Shanghai Medical College, Fudan University, Shanghai 200032, China; ${ }^{2}$ Institute of Biomedical Sciences, Fudan University, 138 Yixue Yuan Road, Shanghai 200032, China; ${ }^{3}$ State Key Laboratory of Genetic Engineering, School of Life Sciences, Fudan University, Shanghai 200433, China

*These two authors contributed equally to this work.

Correspondence: Yanhui Xu

E-mail: xuyh@fudan.edu.cn

\section{References}

1 Sharif J, Muto M, Takebayashi S, et al. The SRA protein Np95 mediates epigenetic inheritance by recruiting Dnmt1 to methylated DNA. Nature 2007; 450:908-912.

2 Bostick M, Kim JK, Esteve PO, et al. UHRF1 plays a role in maintaining DNA methylation in mammalian cells. Science 2007; 317:1760-1764.

3 Karagianni P, Amazit L, Qin J, Wong J. ICBP90, a novel methyl K9 H3 binding protein linking protein ubiquitination with heterochromatin formation. Mol Cell Biol 2008; 28:705-717.

4 Nady N, Lemak A, Walker JR, et al. Recognition of multivalent histone states associated with heterochromatin by UHRF1 protein. J Biol Chem 2011; 286:24300-24311.

5 Peters AH, O'Carroll D, Scherthan H, et al. Loss of the Suv39h histone methyltransferases impairs mammalian heterochromatin and genome stability. Cell 2001; 107:323-337.

6 Zilberman D, Gehring M, Tran RK, Ballinger T, Henikoff S. Genome-wide analysis of Arabidopsis thaliana DNA methylation uncovers an interdependence between methylation and transcription. Nat Genet 2007; 39:61-69.

7 Zhang X, Yazaki J, Sundaresan A, et al. Genome-wide highresolution mapping and functional analysis of DNA methyla- 
tion in Arabidopsis. Cell 2006; 126:1189-1201.

8 Papait R, Pistore C, Grazini U, et al. The PHD domain of Np95 (mUHRF1) is involved in large-scale reorganization of pericentromeric heterochromatin. Mol Biol Cell 2008; 19:3554-3563.

9 Rottach A, Frauer C, Pichler G, et al. The multi-domain pro- tein Np95 connects DNA methylation and histone modification. Nucleic Acids Res 2010; 38:1796-1804.

10 Nady N, Lemak A, Walker JR, et al. Recognition of multivalent histone states associated with heterochromatin by UHRF1. J Biol Chem 2011; 286:24300-24311.

(Supplementary information is linked to the online version of the paper on the Cell Research website.) 